\title{
PROSPECÇÃO CIENTÍFICA SOBRE RESÍDUOS DE SERVIÇOS DE SAÚDE NA ÁREA DAS CIÊNCIAS AMBIENTAIS
}

\author{
Eder Almeida Freire ${ }^{1}$ \\ Maria Rosilene Cândido Moreira ${ }^{2}$
}

\section{RESUMO}

Este estudo se propôs a realizar uma prospecção científica sobre Resíduos de Serviços de Saúde (RSS) nas produções científicas brasileiras da última década na área de Ciências Ambientais (CiAmb). Trata-se de estudo bibliométrico que utilizou como fonte de dados a Biblioteca Científica Eletrônica on line SciELO Brasil, por ser de fácil acesso e difundir de maneira abrangente os artigos publicados no país, e o Banco de Teses da Coordenação de Aperfeiçoamento de Pessoal de Nível Superior, que coordena o Sistema de Pós-graduação brasileiro. Foram encontrados 35 artigos, com predomínio na área das Ciências da Saúde $(65,7 \%)$ e em revistas de alta qualidade científica (62,7\%), além de 100 dissertações e 19 teses, porém com apenas $12,6 \%$ destas classificadas nas CiAmb e concentradas no sul do país $(20,1 \%)$. Evidenciou-se escassez de trabalhos sobre RSS na perspectiva das CiAmb, gerando lacunas nessa seara e obstaculizando novos olhares no campo da sustentabilidade ambiental.

Palavras-chave: Resíduos sólidos. Resíduos de serviços de saúde. Sustentabilidade ambiental.

Recebido em: 30/06/2016 I Aceito em: 20/07/2016

\footnotetext{
${ }^{1}$ Possui mestrado e doutorado em Bioquímica pela Universidade Federal do Ceará. Atualmente é Professor Adjunto IV da Universidade Federal de Campina Grande. Tem experiência na área de Bioquímica, atuando principalmente nos seguintes temas: Proteínas, Lectinas, Bactérias, e Fungos. Orienta o grupo de estudantes do Laboratório de Tecnologias de Informação e Comunicação em Saúde (LATICS-UFCG) - E-mail: ederfreire8@gmail.com

2 Possui graduação em Enfermagem pela Universidade Estadual do Ceará (1998), especialização em Saúde da Família, Saúde Coletiva e Educação em Enfermagem, Mestrado pelo Programa de Pósgraduação em Saúde Coletiva da Universidade Federal de São Paulo - UNIFESP (2010) e Doutorado em Biotecnologia pela RENORBIO/UFPB (2013).É professora Adjunta da Universidade Federal do Cariri (UFCA) - E-mail: rosilene.moreira@ufca.edu.br
} 


\section{INTRODUÇÃO}

Desenvolvimento Sustentável (DS) é conceituado pela Comissão Mundial sobre Meio Ambiente e Desenvolvimento (CMMAD) como sendo aquele capaz de atender às necessidades do mundo contemporâneo, sem comprometer as gerações futuras na satisfação de suas próprias necessidades. Esta definição abrange três dimensões consideradas os pilares do DS, sendo elas a ambiental, a econômica e a social (BARBOSA, 2008).

Com esta compreensão, a Sustentabilidade Ambiental pode ser alcançada através da intensificação dos recursos existentes e potenciais, da limitação do consumo de recursos esgotáveis e da utilização de produtos renováveis, sendo necessário para isso o incremento de pesquisas que proponham o desenvolvimento de tecnologias limpas, pois significa produzir e consumir de maneira a garantir os recursos sem esgotá-los (NASCIMENTO, 2012).

Consistindo uma das principais dimensões, as demandas socioambientais do DS são elementos inerentes às Ciências Ambientais (CiAmb), uma vez que o escopo desta área é multidisciplinar, requerendo a compilação de diferentes áreas do saber, possibilitando reflexões sob diferentes compreensões e perspectivas (COORDENAÇÃO DE APERFEIÇOAMENTO DE PESSOAL DE NÍVEL SUPERIOR - CAPES, 2013).

As CiAmb tornaram-se alvo de estudos mais delineados nos programas de pós-graduação brasileiros a partir da criação formal desta área do conhecimento, no ano de 2011, pelas CAPES, resultante da magnitude dos problemas ambientais multifacetados, cujo olhar multidisciplinar se faz pertinente. Ademais, a complexidade da relação entre os sistemas antrópicos e naturais já despontava como arcabouço inicial nas pesquisas desenvolvidas em alguns programas de pósgraduação do país, que primavam por estudos de natureza multi e interdisciplinares, culminando na criação desta área do conhecimento, que abrange, dentre outros temas, as dimensões do Desenvolvimento Sustentável (CAPES, 2013).

Considerando-se então que o Desenvolvimento Sustentável abriga debates sob variados enfoques, torna-se evidente que a dimensão ambiental do DS encontra espaço nos diversos ambientes sociais e econômicos, envolvendo a participação consciente de todos os atores envolvidos, quer sejam agentes governamentais ou 
não, a fim de buscarem soluções sustentáveis para o manejo dos resíduos gerados nos assentamentos humanos.

Sob este aspecto, evidencia-se que a produção de resíduos esbarra no desafio de saber como gerenciá-los sem que isso ocasione danos a natureza, visto que, há centenas de anos, o homem tem mantido uma relação quase que de dependência com estes. Desde os primórdios de nossa existência o acúmulo de resíduos (mesmo que orgânicos em sua maioria) tornou-se algo inevitável (RIBEIRO, 2011).

Com as revoluções industriais ocorridas na Inglaterra e o conseqüente crescimento desenfreado e desordenado dos aglomerados urbanos e rurais, o equacionamento da geração excessiva e da disposição final ambientalmente segura dos resíduos sólidos despontou como um grande desafio para a humanidade. Uma prioridade maior foi destinada ao tema em uma escala global a partir da Conferência Rio 92, em virtude das discussões sobre a contribuição indireta dos resíduos sólidos para o aquecimento global e as mudanças climáticas (JACOBI; BESEN, 2011).

Ainda nesse sentido, a temática dos resíduos sólidos emerge como um assunto que vem sendo cada vez mais explorado, tanto no âmbito das políticas públicas, como nos ambientes de trabalho e domiciliares, principalmente, devido ao seu potencial de poluição e de reutilização. Nessa perspectiva, dentre os diferentes tipos de resíduos gerados pelo homem, os produzidos nos serviços de saúde têm merecido maior atenção nos últimos anos, especialmente por suas frações infectantes.

Os resíduos sólidos dos serviços de saúde, também denominados lixo hospitalar ou resíduos de serviços de saúde (RSS) podem ser definidos como "rejeitos produzidos pelos estabelecimentos de saúde, abrangendo hospitais, clínicas veterinárias, farmácias, clínicas médicas e odontológicas, laboratórios de análises clínicas e outros (DOI; MOURA, 2011).

Dessa forma, para a segregação desses resíduos, em 2005, o Conselho Nacional de Meio Ambiente (CONAMA) publicou a Resolução no 358, que os classifica em cinco grupos: A, B, C, D e E. Enquadram-se no grupo A os que apresentam risco potencial à saúde pública e ao meio ambiente devido à possível presença de agentes biológicos, dentre eles, materiais contendo secreções e líquidos orgânicos; no grupo $\mathrm{B}$ encontram-se os resíduos químicos; no grupo $\mathrm{C}$, os 
rejeitos radioativos; no grupo $D$, os resíduos comuns e no grupo $E$ os materiais perfurocortantes ou escarificantes, tais como agulhas e escalpes (BRASIL, 2005).

Entretanto, mesmo com essa classificação, alguns estabelecimentos de saúde desconhecem a quantidade e a composição dos resíduos que produzem (REIS et al, 2013), resultando no seu inadequado manejo, oferecendo risco ao ambiente e à saúde. Além disso, observa-se que essas instituições, independente de sua natureza pública ou privada, consomem material de uso único em dimensão excedente, o que encarece seus custos e poluem o meio ambiente, denotando práticas não conscientes do uso dos recursos naturais do planeta.

Nesta perspectiva, as organizações são chamadas a pautar por suas práticas, tanto administrativas quanto operacionais, em preceitos sustentáveis e que não prejudicam ao meio ambiente. Com esta compreensão, entende-se que a sustentabilidade deve desempenhar um papel fundamental nas organizações modernas, embora a divulgação de relatórios de sustentabilidade nas empresas públicas esteja ainda em uma fase embrionária se comparado com as empresas privadas (GARCIA-SANCHEZ; FRÍAS-ACEITUNO; RODRIGUEZ-DOMINGUEZ, 2013).

Como forma de exemplificar esta situação, o uso dos copos descartáveis nos diversos segmentos sociais vem sendo alvo de pesquisas (UTRINI et al., 2007) e, com base nos achados investigativos, são apresentadas medidas inovadoras de redução do seu uso nas empresas que possuem um elevado contingente de trabalhadores, resultando em experiências exitosas (PEREIRA; XAVIER; MORAES, 2007).

Outro estudo sobre desenvolvimento sustentável, realizado em hospitais catalães, revelou que $80 \%$ dos hospitais têm realizado iniciativas para reduzir o consumo de eletricidade, papel, água e climatização. Essas iniciativas implicam em uma economia aproximada de $7 \%$ nos custos das instituições. Outros dados significativos mostram que $52 \%$ dos prestadores consideram que a prática cotidiana já inclui critérios de sustentabilidade e que 30\% dos hospitais têm promovido iniciativas de integração de critérios de sustentabilidade. Outros $25 \%$ dos serviços dispõem ou estão em processos de alcançar uma certificação ambiental (FERNANDES, 2011).

Ademais, os hospitais saudáveis, verdes ou sustentáveis buscam satisfazer a sociedade por meio de edificações que tenham menor impacto ambiental e melhores 
condições em termos de conforto aos seus usuários. Os chamados prédios sustentáveis têm características de ser ambientalmente mais adequados do que os edifícios convencionais. Possuem conceitos da arquitetura bioclimática e uso de novas tecnologias diferenciadas - materiais apropriados, captação/produção de energias limpas (eólica ou solar) e aproveitamento de águas pluviais. Tais hospitais são edificações que lançam mão desses mecanismos para minorar os danos ao meio ambiente.

Associado a isto, promover ações de educação em saúde nos ambientes sociais e de trabalho e concomitante, realizar pesquisas que ofereçam possibilidades de melhoria da qualidade de vida, atribuindo à Universidade a coresponsabilidade na educação ambiental parece ser uma apropriada estratégia, pois, além de visar à formação de uma consciência critica, este tipo de parceria desenvolve uma consciência mais duradoura do papel de cada um na preservação da natureza e da adoção de boas práticas em relação aos recursos naturais.

Nesse sentido, preocupados com a preservação da natureza e na busca de tecnologias renováveis limpas, os pesquisadores brasileiros tem enveredado esforços em pesquisas e produções científicas que discutem amplamente a questão da Sustentabilidade e sua dimensão ambiental, debates expressos em congressos e simpósios da área, nos cursos de pós-graduação e nas produções textuais científicas nacionais e internacionais. Entretanto, poucos são os estudos realizados sobre o que é produzido na área de sustentabilidade ambiental e publicado em periódicos (SOUZA; RIBEIRO, 2013).

Com base nessa constatação, e considerando ser premente que mais estudos aprofundem a temática, uma vez que a questão ambiental sustentável constitui tema inesgotável de investigação e reflexões, propôs-se o presente trabalho, que buscou realizar uma prospecção científica sobre RSS nos artigos publicados em periódicos nacionais e em dissertações e teses oriundas dos programas nacionais de pós-graduação stricto sensu na área de Ciências Ambientais.

Pretende-se, com este artigo, contribuir para estimular reflexões acerca dos RSS na perspectiva das CiAmb, fomentando curiosidades científicas, além de discorrer sobre o que tem sido explorado atualmente nesta dimensão e assim contribuir para o Desenvolvimento Sustentável. 


\section{MATERIAIS E MÉTODOS}

Este estudo configura-se como descritivo, documental, com abordagem quantitativa, que utilizou técnicas de análise bibliométrica, cuja intenção é quantificar e analisar a produção científica acerca do tema escolhido e assim possibilitar a elucidação de estratégias para futuras pesquisas que aprofundem 0 assunto (SOUZA; RIBEIRO, 2013).

Por bibliometria compreende-se um método quantitativo e estatístico de medição de índices de produção/disseminação do conhecimento científico, uma vez que, estudos dessa natureza abrangem toda pesquisa que tem 0 intuito de quantificar os processos de comunicação escrita (PRITCHARD, 1969; ARAÚJO, 2006 apud FERREIRA, 2010). Com esta definição, optou-se por este tipo de estudo, uma vez que ele pretende investigar amplamente a produção textual de artigos científicos, dissertações e teses sobre RSS, enquadrando-se na essência dos estudos bibliométricos.

Para a análise dos trabalhos, foram consideradas as três leis básicas da bibliometria: a Lei de Bradford (produtividade de periódicos), ou Lei da dispersão, sob a qual é possível estabelecer o núcleo e as áreas de dispersão sobre determinado assunto em um mesmo conjunto de revistas, através da medição da produtividade dos periódicos; a Lei de Lotka (produtividade de autores), ou Lei do Quadrado Inverso, considera a perspectiva da probabilidade em publicar trabalhos, considerando que à medida que muitos autores escrevem um só trabalho isto se torna inversamente proporcional a quantidade de autores diversos que escrevem sobre um mesmo tema, ou seja, quanto mais se publica, mais fácil se torna publicar novo trabalho; e c) Lei de Zipf (frequência de ocorrência de palavras) ou Lei do Menor Esforço, que reflete a medição da frequência do aparecimento dos termos pesquisados nos documentos científicos, gerando uma listagem ordenada sobre determinado assunto que viabiliza sua medição e análise (FERREIRA, 2010).

No presente estudo, para cumprir os preceitos da Lei de Bradford, utilizou-se o Banco de Dados do Portal de Revistas Eletrônicas on line SciELO Brasil e o Banco de Teses da CAPES, a fim de identificar os periódicos e as instituições depositárias das produções acerca do tema sob estudo e assim poder verificar a dispersão do tema em âmbito nacional; para cumprir uma das premissas da Lei de Lotka, buscouse averiguar as publicações e classificá-las conforme as diversas áreas do 
conhecimento, tomando como base as credenciais dos autores informadas nos artigos. Por último, em cumprimento à Lei de Zipf, foi adotado um termo de busca para identificação dos trabalhos - Resíduos de serviços de saúde - a fim de padronizar as pesquisas e encontrar o maior número de publicações e produções acadêmicas existentes nas bibliotecas investigadas.

Foram analisados artigos publicados na última década (2005 - 2015) e dissertações e teses defendidas nos últimos cinco anos (2010 - 2015), a fim de estabelecer uma análise de tendência. Os artigos foram lidos na íntegra e avaliados quanto ao periódico de publicação, área de conhecimento relacionada, conteúdo da pesquisa, formação/credenciais dos autores e evolução cronológica das publicações.

Além desses quesitos, o conceito Qualis da CAPES também foi mensurado, tomando-se por base a avaliação trienal 2013 (CAPES, 2013) e a área de conhecimento CiAmb na definição dos estratos, pois considera-se que, para a realidade brasileira, o Qualis da CAPES e o formato de avaliação por estratos é a melhor maneira de analisar a qualidade das revistas científicas (FERREIRA, 2010).

As dissertações e teses tiveram seus resumos lidos e foram examinadas quanto à área de conhecimento envolvida, conteúdo do trabalho, ano de defesa, instituição de educação superior vinculada e evolução cronológica das produções.

Todos os documentos textuais foram lidos durante o mês de agosto de $2015 \mathrm{e}$ os resultados digitados e tabulados no Microsoft Excel 2007, sendo apresentados sob a forma de tabelas e gráficos, mediante análise estatística descritiva.

\section{RESULTADOS E DISCUSSÃO}

Foram encontrados 35 artigos em 16 periódicos, agrupados conforme a qualificação Qualis CAPES (Tabela 1).

Analisando-se os estratos Qualis, verifica-se que quatro periódicos estão classificados com Qualis A2 na área das CiAmb, representando 51,2\% dos artigos publicados nessa temática (18 artigos), sendo considerado padrão ouro na produção nacional conforme a classificação da CAPES na avaliação dos orientadores pertencentes aos programas de pós-graduação stricto sensu no país. No estrato B1, foram encontrados dois periódicos, com três artigos neles publicados (8,6\%) e no B2 
uma revista $(2,9 \%)$, todos considerados bons estratos para credenciamento, manutenção e qualificação dos programas de mestrado e doutorado no país.

Tabela 1. Publicações sobre RSS, por periódico e classificação Qualis em CiAmb.

\begin{tabular}{lrrc}
\hline \multicolumn{1}{c}{ Periódico } & \multicolumn{2}{c}{$\mathbf{N}^{\circ}$ de artigos } & Estrato \\
& \multicolumn{1}{c}{$\%$} & Qualis \\
Eng. Sanit. Ambient. & 10 & 28,5 & A2 \\
Ciênc. saúde coletiva & 04 & 11,3 & A2 \\
Cad. Saúde Pública & 03 & 8,5 & A2 \\
Rev. Bras. Enferm. & 03 & 8,5 & Sem Qualis \\
J. Bras. Patol. Med. Lab. & 02 & 5,7 & Sem Qualis \\
Rev Panam Salud Publica & 02 & 5,7 & B1 \\
Rev. Gaúcha Enferm. & 02 & 5,7 & Sem Qualis \\
Acta paul. enferm. & 01 & 2,9 & Sem Qualis \\
Arq Bras Endocrinol Metab. & 01 & 2,9 & Sem Qualis \\
Interface (Botucatu) & 01 & 2,9 & Sem Qualis \\
Quím. Nova & 01 & 2,9 & A2 \\
Rev. Latino-Am. Enfermagem & 01 & 2,9 & Sem Qualis \\
Rev. Soc. Bras. Med. Trop. & 01 & 2,9 & B1 \\
Rev. bras. saúde ocup. & 01 & 2,9 & B4 \\
Rev. bras. saúde prod. anim. & 01 & 2,9 & B2 \\
Texto contexto - enferm. & 01 & 2,9 & Sem Qualis \\
TOTAL & 35 & 100,0 & - \\
\hline Fonte: Autian
\end{tabular}

Fonte: Autoria própria, 2015.

Entretanto, em um elevado número de periódicos (08 revistas) não foi encontrada classificação Qualis para a área de CiAmb, representando 34,4\% (12 artigos) da produção encontrada em RSS. Associado a isto, observa-se que cinco destes periódicos são específicos da área de Enfermagem, cujas publicações poderiam ser agregadas, contribuindo para aumentar o arsenal de publicações em CiAmb e assim reforçar o caráter interdisciplinar desta área de conhecimento.

Destaca-se que a área de CiAmb foi introduzida no contexto da pósgraduação da CAPES no ano de 2011, decorrente da necessidade de contemplar as produções que envolvem a complexidade dos problemas ambientais, mediante o caráter indissociável entre os sistemas antrópicos e naturais que emergem no mundo contemporâneo (CAPES, 2013).

Analisando-se a evolução cronológica das publicações, verifica-se que há uma tendência crescente, porém discreta, com picos pontuais nos anos de $2008 \mathrm{e}$ 2011, sendo de 2,9 a média de artigos publicados por ano sobre a temática em questão, durante a década pesquisada (Figura 1). 


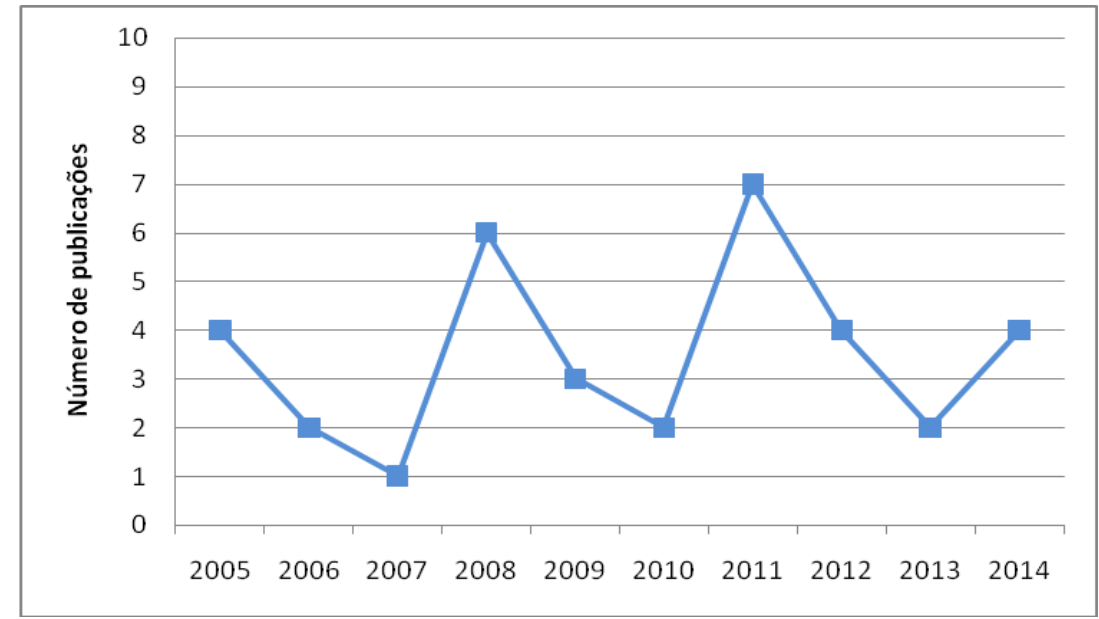

Figura 1. Evolução cronológica das publicações sobre RSS.

Fonte: Autoria própria, 2015.

Quanto às áreas de conhecimento relacionadas às publicações (Tabela 2), verifica-se que as Ciências da Saúde agruparam a maior parte das publicações (65,7\%), enfocando, sobretudo, conhecimento de RSS no âmbito universitário (MORESCHI et. al, 2014; SHINZATO et al., 2010); manejo de RSS nos estabelecimentos de atenção primária (SANTOS; SOUZA, 2012; ALVES et al., 2012), secundária (PEREIRA et al, 2013), terciária (RAMOS et al, 2011; DOI; MOURA, 2011) e em laboratórios de análises clínicas (ALMEIDA et al., 2011; GONÇALVES et al, 2011) e vulnerabilidade dos trabalhadores que manipulam esses resíduos (LAZZARI; REIS, 2011).

As Engenharias também concentraram produção na temática, ocupando o segundo lugar em maior quantidade de artigos publicados $(28,5 \%)$, revelando pesquisas sobre o gerenciamento dos RSS pelos órgãos governamentais municipais (SILVA; SPERLING; BARROS, 2014; GOMES; ESTEVES, 2012;); classificação dos RSS gerados em serviços hospitalares (ADUAN et al, 2014) e caracterização microbiológica existente em RSS (SILVA et al., 2011a; SILVA et al., 2011b).

Tabela 2. Artigos sobre RSS classificados por área temática.

\begin{tabular}{lcr}
\hline \multicolumn{1}{c}{ Área de conhecimento } & $\boldsymbol{f}$ & \multicolumn{1}{c}{$\%$} \\
Ciências da Saúde & 23 & 65,7 \\
Engenharias & 10 & 28,5 \\
Ciências Agrárias & 01 & 2,9 \\
Ciências Exatas e da Terra & 01 & 2,9 \\
TOTAL & 35 & 100,0 \\
\hline
\end{tabular}

Fonte: Autoria própria, 2015. 
Destacam-se as áreas de Ciências Agrárias, que publicou artigo de pesquisa investigando o gerenciamento de RSS em estabelecimentos veterinários (REIS et al., 2013) e das Ciências Exatas e da Terra, que publicou uma pesquisa caracterizando as cinzas geradas na incineração dos RSS (SILVA; LANGE, 2008). Estas iniciativas demonstram que o tema é complexo e multifacetado, podendo ser matéria de estudo de variadas áreas de conhecimento.

Ressalta-se a ausência da classificação dos artigos na área das CiAmb, embora tenham sido encontrados estudos que se enquadrassem nessa seara do conhecimento.

Em relação à pós-graduação, a análise bibliométrica realizada nos trabalhos encontrados foi subdividida em três tópicos: tipo de trabalho (dissertação ou tese), instituições de educação depositárias e evolução das produções.

Embora verificando que no portal da CAPES só estão disponíveis os trabalhos defendidos nos anos de 2011 e 2012, inicialmente efetuou-se a busca das produções pelo termo Sustentabilidade Ambiental, sendo encontrado um total de 1.630 registros; entretanto, refazendo-se a pesquisa com a substituição do termo da busca por Resíduos de Serviços de Saúde, resultaram 119 trabalhos, sendo 100 oriundos dos programas de mestrado e 19 de doutorado (Tabela 3).

Tabela 3. Dissertações e Teses sobre Sustentabilidade Ambiental e RSS

\begin{tabular}{lrr}
\multicolumn{1}{c}{ Descrição } & \multicolumn{1}{c}{$f$} & $\%$ \\
\hline Termo da busca: Sustentabiliade Ambiental & 1.117 & 68,5 \\
Dissertação (mestrado acadêmico) & 191 & 11,7 \\
Dissertação (mestrado profissional) & 306 & 18,8 \\
Tese (doutorado) & 1.630 & 100,0 \\
TOTAL & 81 & 68,0 \\
Termo da busca: Resíduos de Serviços de Saúde & 19 & 16,0 \\
Dissertação (mestrado acadêmico) & 19 & 16,0 \\
Dissertação (mestrado profissional) & 119 & 100,0 \\
Tese (doutorado) & & \\
TOTAL & &
\end{tabular}

Fonte: Autoria própria, 2015.

Avaliando-se os tipos de produção científica, observa-se que as publicações sobre RSS foram produzidas, em sua maioria, a partir dos cursos de mestrado, haja vista que as dissertações representaram $84 \%$ do total de produções, com 81 (68\%) de programas de mestrado acadêmico e 19 (16\%) de mestrado profissional, sinalizando a intensa contribuição desses programas para o incremento das 
pesquisas em RSS no país, embora tais trabalhos ainda sejam incipientes quando comparados ao montante de trabalhos na temática maior, pois este tipo de pesquisa representa somente $7,3 \%$ do total de produções encontradas.

Este dado reflete uma extensa lacuna, haja vista haver incipiente exploração deste campo do conhecimento, resultando em escassez de revelações científicas, inovações e incremento de práticas sustentáveis nos ambientes relacionados à saúde.

Além disso, a maior quantidade de produções sobre RSS concentra-se nas Universidades Estaduais de Maringá e do Oeste do Paraná, somando 20,1\% do total de trabalhos defendidos (Tabela 4), embora tenham sido verificados trabalhos cadastrados em instituições de educação superior de outros estados brasileiros.

Tabela 4. Trabalhos sobre RSS por IES depositárias.

\begin{tabular}{lrr}
\hline \multicolumn{1}{c}{ IES } & $\boldsymbol{f}$ & \multicolumn{1}{c}{$\%$} \\
Universidade Estadual de Maringá & 14 & 11,7 \\
Univ. Estadual do Oeste do Paraná & 10 & 8,4 \\
Fundação Oswaldo Cruz & 07 & 5,9 \\
Universidade Estadual de Campinas & 04 & 3,4 \\
Universidade de São Paulo & 04 & 3,4 \\
Universidade Federal de Goiás & 04 & 3,4 \\
Demais IES & 76 & 63,8 \\
TOTAL & 119 & 100,0 \\
\hline
\end{tabular}

Fonte: Autoria própria, 2015.

Para analisar a evolução cronológica das produções acadêmicas, analisou-se o quantitativo de trabalhos defendidos no biênio 2011-2012. Entretanto, embora a análise dos dois anos disponíveis não possibilite traçar um panorama sobre a evolução cronológica das produções, os dados apresentados nesses anos registraram o quantitativo de 62 trabalhos em 2011 e 57 em 2012, sinalizando um decréscimo de um ano para o outro.

Além disso, esses dados refletem uma baixa produção textual oriunda dos programas de pós-graduação do país, cuja média de dissertações foi de apenas 50 e de teses 9,5, por ano, durante o biênio pesquisado.

Analisando-se as produções acadêmicas conforme a área de conhecimento relacionada, verifica-se que a maior parte delas está concentrada nas Engenharias, que detém $44,5 \%$ das produções acadêmicas sobre RSS e em segundo lugar 
aparecem as CiAmb, representando $12,6 \%$ dos trabalhos defendidos no período estudado (Tabela 5).

Tabela 5. Áreas de conhecimento relacionadas aos trabalhos acadêmicos sobre RSS.

\begin{tabular}{lcr}
\hline \multicolumn{1}{c}{ Área de conhecimento } & $\boldsymbol{f}$ & $\%$ \\
Engenharias & 53 & 44,5 \\
Ciências Ambientais & 15 & 12,6 \\
Saúde Pública/Saúde Coletiva & 14 & 11,8 \\
Enfermagem & 04 & 3,4 \\
Administração & 03 & 2,5 \\
Medicina Veterinária & 03 & 2,5 \\
Demais áreas & 27 & 22,7 \\
TOTAL & 119 & 100,0 \\
\hline
\end{tabular}

Fonte: Autoria própria, 2015.

Foram identificados ainda trabalhos acadêmicos nas áreas de Enfermagem, Administração, Medicina Veterinária, Direito e outras, em uma demonstração de que os RSS constituem assunto transversal para as diversas áreas do conhecimento científico.

Ressalta-se a existência de poucos trabalhos defendidos sobre RSS relacionando-os às CiAmb; embora tenham sido encontrados estes estudos, eles representam somente $12,6 \%$ da produção total de dissertações e teses defendidas no mesmo período.

\section{CONCLUSÕES}

A análise bibliométrica das produções científicas nacionais sobre RSS revelou que este tema vem sendo estudado em diversas áreas do conhecimento, com discreta tendência crescente nos artigos, mas decrescente nos trabalhos oriundos da pós-graduação, embora, tenham sido encontrados mais trabalhos defendidos do que artigos publicados, sinalizando a necessidade de mais publicações para agregar valor aos cursos de mestrado e doutorado, pesquisadores envolvidos e instituições depositárias.

Sob outro enfoque, evidenciou-se escassez de trabalhos sobre RSS na perspectiva das Ciências Ambientais, sinalizando haver uma lacuna nesta seara do conhecimento, necessitando que estudos futuros teçam reflexões acerca dos possíveis fatores contribuintes para este reduzido número de trabalhos envolvendo essa interface. 


\title{
SCIENTIFIC PROSPECTION ABOUT MEDICAL WASTE IN THE AREA OF ENVIRONMENTAL SCIENCES
}

\begin{abstract}
This work aimed to conduct scientific prospection about Medical Wast (MW) in Brazilian scientific productions of the last decade in the area of Environmental Sciences (ES). It is bibliometric study using as a data source the Scientific Electronic Library online SciELO Brazil, being easy to access and disseminate comprehensive way the articles published in the country and the Bank Thesis Level Personnel Training Coordination Superior, which coordinates the Brazilian Postgraduate System. Thirty five articles were found, predominantly in the area of Health Sciences (65.7\%) and in high quality scientific journals (62.7\%), and one hundred dissertations and nineteen theses, but with only $12.6 \%$ of these classified in ES and concentrated in the south of the country $(20.1 \%)$. It revealed a shortage of publications on $\mathrm{MW}$ in view of ES, creating gaps that harvest and hindering new perspectives in the field of environmental sustainability.
\end{abstract}

Keywords: Solid wast; Medical wast; Environmental Sustainability.

\section{REFERÊNCIAS}

ADUAN, S.A. et al . Avaliação dos resíduos de serviços de saúde do Grupo A em hospitais de Vitória (ES), Brasil. Eng. Sanit. Ambient., Rio de Janeiro, v. 19, n. 2, p. 133-141, 2014.

ALMEIDA, M.C.A. et al. Concentração de fenol em resíduos de laboratórios de análises clínicas. J. Bras. Patol. Med. Lab.; v.47, n.4, p. 431-437, 2011.

ALVES, S.B. et al. Manejo de resíduos gerados na assistência domiciliar pela Estratégia de Saúde da Familia. Rev. Bras. Enferm., v.65, v.1, p.128-134, 2012.

BARBOSA, G.S. O desafio do desenvolvimento sustentável. Revista Visões, v.1, n.4, 2008.

BRASIL. Ministério do Meio Ambiente. Conselho Nacional do Meio Ambiente (CONAMA). Resolução 358 - dispõe sobre o tratamento e a disposição final dos resíduos dos serviços de saúde e dá outras providências. Brasília, 2005. Disponível em: <http://www.mma.gov.br/port/conama/res/res05/res35805.pdf>. Acesso em: 02 ago. 2015.

COORDENAÇÃO DE APERFEIÇOAMENTO DE PESSOAL DE NÍVEL SUPERIOR (CAPES). Avaliação trienal 2013. Disponível em: 
<http://qualis.capes.gov.br/webqualis/publico/documentosDeArea.seam?conversatio nPropagation=begin>. Acesso em: 20 jul. 2015.

DOI, K. M.; MOURA, G. M. M. S. S. Resíduos sólidos de serviços de saúde: uma fotografia do comprometimento da equipe de enfermagem. Rev. Gaúcha Enferm., vol.32, n.2, p. 338-344, 2011.

FERREIRA, A.G.C. Bibliometria na avaliação de periódicos científicos.

DataGramaZero - Revista de Ciência da Informação, v.11, n.3, 2010. Disponível em: <http://www.dgz.org.br/jun10/Art_05.htm\#NOTAS>. Acesso em: 20 ago. 2015.

FERNANDES, G. Federação dos Hospitais e Estabelecimentos de Saúde do Estado do Rio de Janeiro (FEHERJ) (Org.). A hora dos hospitáveis verdes e sustentáveis. 2011. Disponível em:

<http://www.feherj.com.br/RevistaSaudeRio/RevistaSaudeRio01.pdf>. Acesso em: 21 set 2015.

GARCIA-SANCHÉZ, I. M.; FRÍAS-ACEITUNO, J. V.; RODRIGUEZ-DOMINGUEZ, L. Determinants of corporate social disclosure in Spanish local governments. Journal of Cleaner Production, v. 39, p.60-72, 2013.

GONÇALVES, E.M.N. et al. Modelo de implantação de plano de gerenciamento de resíduos no laboratório clínico. J. Bras. Patol. Med. Lab., v. 47, n.3, p. 249-255, 2011.

GOMES, L. P.; ESTEVES, R. V. R. Análise do sistema de gerenciamento dos resíduos de serviços de saúde nos municípios da bacia hidrográfica do Rio dos Sinos, Rio Grande do Sul, Brasil. Eng. Sanit. Ambient., Rio de Janeiro, v. 17, n. 4, p. 377-384, 2012 .

JACOBI, P.R., BESEN, G.R. Solid waste management in São Paulo: the challenges of sustainability. Estudos Avançados. São Paulo, vol. 25, n. 71, p. 135-158, 2011.

LAZZARI, M.A.; REIS, C.B. Os coletores de lixo urbano no município de Dourados (MS) e sua percepção sobre os riscos biológicos em seu processo de trabalho.

Ciênc. saúde coletiva, v.16, n.8, p.3437-3442, 2011.

MORESCHI, Claudete et al . A importância dos resíduos de serviços de saúde para docentes, discentes e egressos da área da saúde. Rev. Gaúcha Enferm., Porto Alegre, v. 35, n. 2, p. 20-26, 2014. 
NASCIMENTO, E.P. Trajetória da sustentabilidade: do ambiental ao social, do social ao econômico. Estudos Avançados, v.26, n. 74, p. 51-64, 2012.

PEREIRA, M.S. et al. Gerenciamento de resíduos em unidades não hospitalares de urgência e emergência. Rev. Latino-Am. Enfermagem, v.21, n.especial, p.259-266, 2013.

PEREIRA, A.C.S.G.; XAVIER, M.P.; MORAES, H.S. Redução no Consumo de Copos Descartáveis no Serviço de Atendimento Unimed - Goiânia - GO Brasil. Estudo de Caso. 2014. Disponível em:

<http://www.hospitaissaudaveis.org/arquivos/Unimed\%20Goiania_Residuos.pdf>. Acesso em: 04 ago. 2015.

RAMOS, Y.Z. et al. Vulnerabilidade no manejo dos resíduos de serviços de saúde de João Pessoa (PB, Brasil). Ciênc. saúde coletiva, v. 16, n. 8, p. 3553-3560, 2011.

REIS, M. A. et al. Conhecimento, prática e percepção sobre o gerenciamento de resíduos de serviços de saúde em estabelecimentos médicos veterinários de Salvador, Bahia. Rev. bras. saúde prod. anim., v.14, n.2, p.287-298, 2013.

RIBEIRO, J. C. J. Seminário Internacional sobre resíduos de Equipamentos eletroeletrônicos. Belo Horizonte: FEAM, 22 fev. 2011.

SANTOS, M.A.; SOUZA, A.O. Conhecimento de enfermeiros da Estratégia Saúde da Família sobre resíduos dos serviços de saúde. Rev. Bras. Enferm., v.65, n.4, p.645652, 2012.

SHINZATO, M.P. et al. Análise preliminar de riscos sobre o gerenciamento dos resíduos de serviços de saúde de uma instituição de ensino em Mato Grosso do Sul: estudo de caso. Rev. bras. saúde ocup., v.35, n.122, p.340-352, 2010.

SILVA, M.L.; LANGE, L.C. Caracterização das cinzas de incineração de resíduos industriais e de serviços de saúde. Quím. Nova, v.31, n.2, p.199-203, 2008.

SILVA, D. F.; SPERLING, E. V.; BARROS, R. T. V. Avaliação do gerenciamento dos resíduos de serviços de saúde em municípios da região metropolitana de Belo Horizonte (Brasil). Eng. Sanit. Ambient., Rio de Janeiro, v. 19, n. 3, p. 251 262, 2014. 
SILVA, C.A.M.C. et al. Caracterização microbiológica de lixiviados gerados por resíduos sólidos domiciliares e de serviços de saúde da cidade do Rio de Janeiro. Eng. Sanit. Ambient., v.16, n.2, p.127-132, 2011a.

SILVA, W.S. et al. Avaliação dos benefícios da coleta de lixo em Palmas, Tocantins: uma aplicação do método de avaliação contingente. Eng. Sanit. Ambient., v.16, n.2, p.141-148, 2011b.

SOUZA, M.T.S.; RIBEIRO, H.C.M. Sustentabilidade ambiental: uma meta-análise da produção brasileira em periódicos de administração. Revista de Administração contemporânea, v.17, n.3, p.368-396, 2013.

UTRINI, D.P. et al. Análise de viabilidade da substituição dos copos descartáveis por copos de vidros na cantina fem, UNICAMP. Revista Ciências do Ambiente OnLine, v. 3, n.1, p.75-76, 2007. 